\title{
NOTA
}

\section{Depredación del sapo gigante (Rhinella horribilis) (Anura: Bufonidae) por el grisón (Galictis vittata) (Carnivora: Mustelidae)}

\author{
Raquel Bone-Guzmán ${ }^{1}$ (D) Javier Carazo Salazar ${ }^{2}$ iD \\ 1. Universidad de Costa Rica, Escuela de Biología, San Pedro, San José, Costa Rica; raquelbone@gmail.com \\ 2. Sociedad Mesoamericana para la Biología y la Conservación, Capítulo Costa Rica, Apartado 91-3100, Santo \\ Domingo, Heredia, Costa Rica; carazo.javier@gmail.com
}

\author{
Recibido 14-V-2021 • Corregido 07-VI-2021 • Aceptado 22-VI-2021
}

DOI: https://doi.org/10.22458/urj.v13i2.3524

\begin{abstract}
Predation of a giant toad (Rhinella horribilis) (Anura: Bufonidae) by the greater grison (Galictis vittata) (Carnivora: Mustelidae)". Introduction: There is little ecological information on the grison, Galictis vittata, including its geographic distribution, diet and habitat. Objective: To report the first record of this grison eating a giant toad. Methods: We examined camera trap images from January 2019 to May 2020. Results: We obtained a photo of a grison with a giant toad in its mouth, from an oil palm plantation next to a regenerating forest in Golfo Dulce, Costa Rica, and assumed a successful act of predation. Conclusions: This record represents the first predation event of $R$. horribilis by $G$. vittata, as well as the first record of a grison in an oil palm plantation in Costa Rica.
\end{abstract}

Keywords: Camera trapping, diet, mustelid, oil palm, Osa Peninsula.
RESUMEN. Introducción: Existe poca información ecológica sobre el tejón o grisón mayor, Galictis vittata, incluida su distribución geográfica, dieta y hábitat. Objetivo: Informar el primer registro de este tejón capturando un sapo gigante. Métodos: Examinamos imágenes de cámaras-trampa de enero de 2019 a mayo de 2020. Resultados: Obtuvimos una foto de un tejón con el sapo en su hocico, en una finca de palma aceitera junto a un bosque en regeneración en Golfo Dulce, Costa Rica, y supusimos que la depredación fue exitosa. Conclusiones: Este registro representa el primer evento de depredación de $R$. horribilis por G. vittata, así como el primer registro de este tejón en una finca de palma aceitera en Costa Rica.

Palabras clave: Fototrampeo, dieta, musélido, palma aceitera, Península de Osa.

El tejón, grisón o hurón grande (Galictis vittata) es una de las especies de mustélidos menos conocidas de América (Bornholdt et al., 2013), de la cual se presentan importantes vacíos de información en cuanto a su ecología a lo largo de su distribución. La especie se encuentra en bajas densidades principalmente en bosques caducifolios y perennifolios, maduros y en regeneración desde el sur de México hasta el noreste de Argentina y el sur de Brasil (Arita et al., 1990; Yesen \& Tarifa, 2003; Cuarón et al., 2016). También se ha reportado en hábitats con distinto grado de perturbación como plantaciones de café, banano, pastizales y otros cultivos (Gallina et al., 1996; Gaudrain \& Harvey, 2003; de la Torre et al., 2009; Herra \& Castañeda, 2014; Meza-Joya et al., 2018; González-Maya et al., 2019).

En Costa Rica se considera una especie rara y difícil de observar, siendo la información disponible sobre su distribución, ecología y estado de conservación escasa y limitada (Timm et al., 1989; Malavassi, 1991; Rodríguez \& Chinchilla, 1996; de la Rosa \& Nocke, 2000; Gaudrain \& Harvey, 
2003; Sáenz-Bolaños et al., 2009; Artavia, 2018). Su estado de conservación a nivel global es de Preocupación Menor (LC) según la Lista Roja de Especies Amenazadas de la UICN (Cuarón et al., 2016), sin embargo, para Costa Rica se encuentra dentro de la categoría de especies de fauna con poblaciones reducidas y amenazadas (Sistema Nacional de Áreas de Conservación [SINAC], 2017).

Su dieta es poco conocida, alimentándose principalmente de vertebrados pequeños y medianos incluyendo roedores, marsupiales, y aves, y en menor cantidad de reptiles (serpientes y lagartijas), anfibios y peces. Ocasionalmente también consumen frutos, artrópodos, y huevos (Sunquist et al., 1989; Kays, 1996; Yesen \& Tarifa, 2003; Hidalgo-Mihart et al., 2018). En cuanto a los anfibios únicamente se reporta el consumo de una especie de Mannophryne (Arobatidae) en Venezuela (Bisbal, 1986) y de Rhinella marina (Bufonidae) en Brasil (Cintra, 1988), no hay informes detallados de su alimentación en Costa Rica.

Como parte de un estudio para determinar el efecto de factores antropogénicos y del paisaje sobre la distribución y conservación de los mamíferos terrestres en la Reserva Forestal Golfo Dulce en la Península de Osa, Costa Rica (Bone-Guzmán \& Chacón-Madrigal 2020), se colocaron 72 estaciones rotativas de cámaras trampa (modelos Bushnell Trophy Cam, Browning Pro y Stealth Cam Strike Force) de enero del 2019 a mayo del 2020 para un total de 6480 noches-trampa siguiendo el protocolo sugerido por Hurtado y Soto (2017).

El 03 de marzo del 2019, a las 13:22 se registró una fotocaptura de un individuo de grisón (G. vittata) con una presa en su hocico identificada como un sapo gigante ( $R$. horribilis; Bufonidae) (Fig. 1) según sus características morfológicas, tamaño y distribución geográfica (Hawley 2016; Leenders 2016). En la imagen el grisón se movilizaba desde una finca de palma aceitera (Elaeis guineensis) hacia el borde de un bosque en regeneración en el sector de San Juan de Sierpe, dentro de la RFGD (coordenadas $08^{\circ} 45^{\prime} 21.16^{\prime \prime} \mathrm{N}$ y 8330'19.08" O, altitud 33 m.s.n.m.) (Fig. 2).

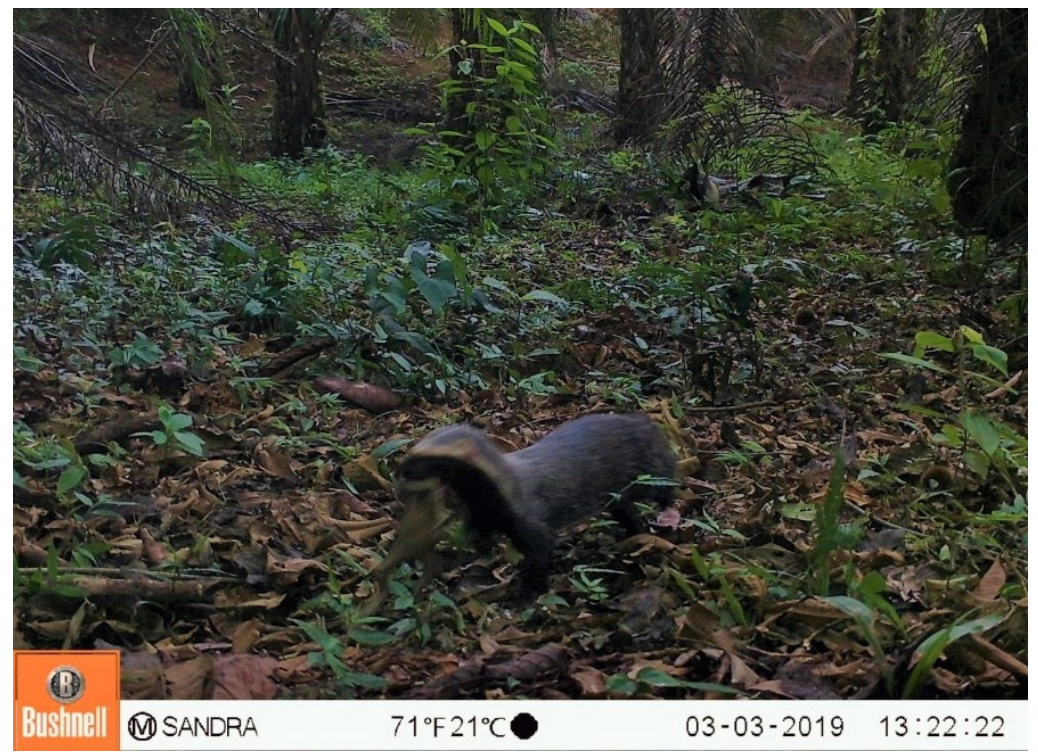

Fig. 1. Fotocaptura de grisón (Galictis vittata) llevando en su hocico un sapo gigante (Rhinella horribilis) en una plantación de palma aceitera en la Reserva Forestal Golfo Dulce, Península de Osa, Costa Rica.

Este es el primer informe de depredación de R. horribilis por G. vittata, así como el primer registro de la especie en fincas de palma aceitera en Costa Rica; coincidiendo con otro caso en Colombia (Meza-Joya et al. 2018). Por esta razón es importante continuar la investigación y monitoreo de estos elusivos mustélidos, así como garantizar la protección y conectividad de sus ecosistemas; tanto hábitats silvestres y áreas agrícolas como los presentes en esta reserva, en donde 
se permiten distintos usos del suelo compatibles con los objetivos de conservación y desarrollo local (Consejo Nacional de Áreas de Conservación [CONAC], 2019).

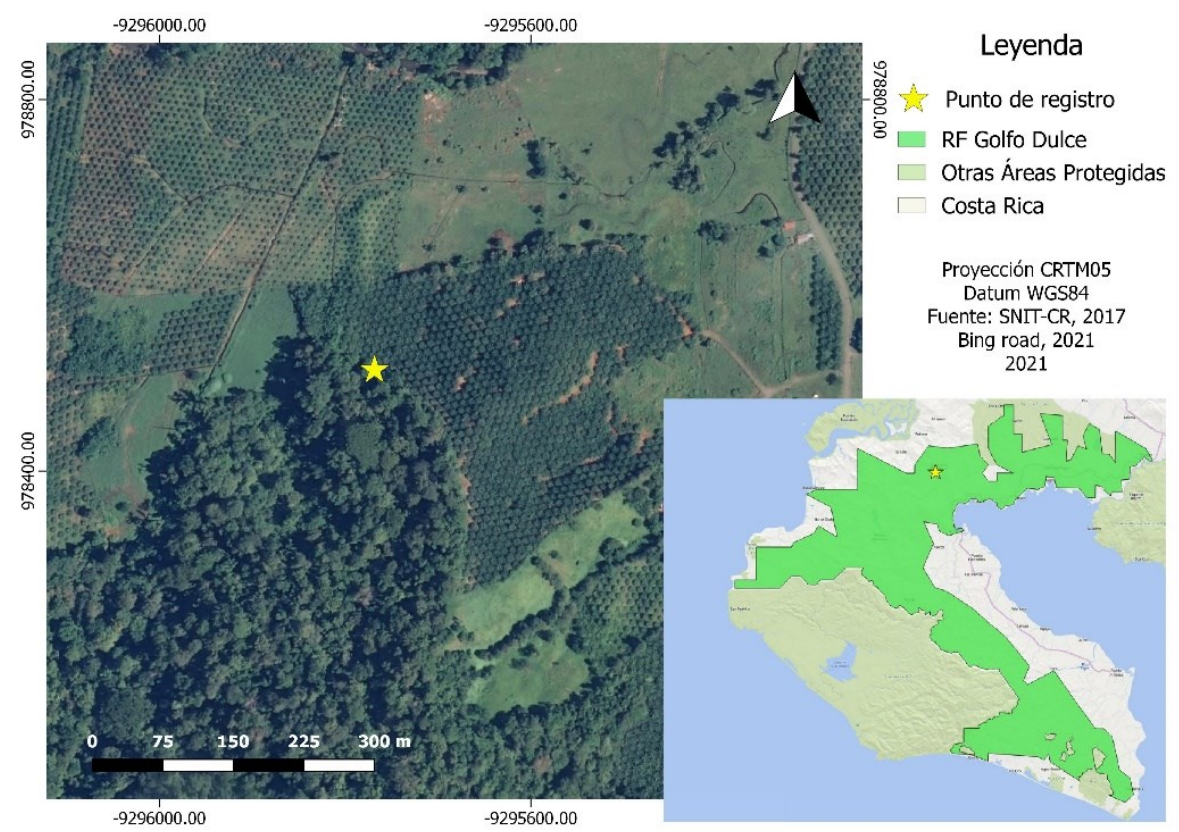

Fig. 2. Ubicación geográfica del registro de grisón (Galictis vittata) llevando en su hocico un sapo gigante (Rhinella horribilis) en una plantación de palma aceitera en la Reserva Forestal Golfo Dulce, Península de Osa, Costa Rica.

\section{AGRADECIMIENTOS}

Un especial agradecimiento a los propietarios de la finca, Sandra González y Douglas Valverde, y a todas las personas de la comunidad de San Juan de Sierpe que luchan por proteger la naturaleza. A los profesores Eduardo Chacón, Reinaldo Aguilar, Jorge Lobo y Guido Saborío por su guía y apoyo. A Josimar Estrella por la corroboración en la identificación del anfibio y a Alexa Morales por la elaboración del mapa. A la Universidad de Costa Rica, Sede Central, del Sur y al Programa Institucional Osa-Golfo Dulce. Este estudio forma parte del proyecto de investigación Pry01-20192019 de la Vicerrectoría de Investigación de la Universidad de Costa Rica, permiso INV-ACOSA-00119 del Área de Conservación Osa - Sistema Nacional de Áreas de Conservación (SINAC). Realizado gracias al apoyo de Rufford Foundation, The Wild Felid Research \& Management Association, SINAC, Fondo de Biodiversidad Sostenible, FONAFIFO y The Nature Conservancy.

\section{ÉTICA, CONFLICTO DE INTERESES Y DECLARACIÓN DE FINANCIAMIENTO}

Los autores declaran haber cumplido con todos los requisitos éticos y legales pertinentes, tanto durante el estudio como en el manuscrito; que no hay conflictos de interés de ningún tipo, y que todas las fuentes financieras se detallan plena y claramente en la sección de agradecimientos. Asimismo, están de acuerdo con la versión editada final del documento. El respectivo documento legal firmado se encuentra en los archivos de la revista.

La declaración de la contribución de cada autor al manuscrito es la siguiente: R.B.G.: Colecta de datos, redacción y revisión del manuscrito. J.C.S.: Análisis de datos, redacción y revisión del manuscrito. 


\section{REFERENCIAS}

Arita, H. T., Robinson, J. G., \& Redford, K. H. (1990). Rarity in neotropical forest mammals and its ecological correlates. Conservation Biology, 4(2), 181-192. https://doi.org/10.1111/j.1523-1739.1990.tb00107.x

Artavia, A. (2018). Estudio con cámaras trampa en Costa Rica: más de dos décadas generando información sobre fauna silvestre. En Sistema Nacional de Áreas de Conservación (Eds.), Uso de cámaras trampa en Costa Rica y sus aplicaciones para el manejo y conservación de la vida silvestre (pp. 35-50). San José, Costa Rica: Proyecto MAPCOBIO-SINAC.

Bisbal, F. J. (1986). Food habits of some Neotropical carnivores in Venezuela (Mammalia, Carnivora). Mammalia, 50(3), 329 - 340. https://doi.org/10.1515/mamm.1986.50.3.329

Bone-Guzmán, R. \& Chacón-Madrigal, E. (2020). From jaguars to margays: spatial distribution and conservation of five feline endangered species and their prey in Golfo Dulce Forest Reserve, Costa Rica. Wild Felid Monitor, 13(2), 21- 22.

Bornholdt, R., Helgen, K., Koepfli, K. P., Oliveira, L., Lucherini, M., \& Eizirik, E. (2013). Taxonomic revision of the genus Galictis (Carnivora: Mustelidae): species delimitation, morphological diagnosis, and refined mapping of geographical distribution. Zoological Journal of the Linnean Society, 167(3), 449-472. https://doi.org/10.1111/j.1096-3642.2012.00859.x

Cintra, R. (1988). Bufo marinus (marine toad) predation. Herpetology Review, 19(4), 82.

Consejo Nacional de Áreas de Conservación [CONAC]. (2019). R-SINAC-CONAC-033-2019. Plan General de Manejo de la Reserva Forestal Golfo Dulce. https://bit.ly/3znb3IL

Cuarón, A.D., Reid, F., González-Maya, J.F. \& Helgen, K. (2016). Galictis vittata. The IUCN Red List of Threatened Species 2016: e.T41640A45211961 https://dx.doi.org/10.2305/IUCN.UK.2016-1.RLTS.T41640A45211961.en

de la Rosa, C.L., \& Nocke, C.C. (2000). A Guide to the Carnivores of Central America: Natural History, Ecology, and Conservation. University of Texas Press.

de La Torre, A., Muench, C., \& Arteaga, M. (2009). Nuevos registros de Grisón Gallictis vittata para la selva Lancandona, Chiapas, México. Revista Mexicana de Mastozoología, 13(1), $109 \quad$ - 114 http://dx.doi.org/10.22201/ie.20074484e.2009.13.1.40

Gallina, S., Mandujano, S. \& González-Romero, A. (1996). Conservation of mammalian biodiversity in coffee plantations of central Veracruz, Mexico. Agroforestry Systems, 33(1), 13-27. https://doi.org/10.1007/BF00122886

Gaudrain, C. \& Harvey, C. (2003). Caza y diversidad faunística en paisajes fragmentados del territorio indígena Bribri de Talamanca, Costa Rica. Agroforestería en las Américas, 10(37), 46-51.

González-Maya, J.F., Chacón, J.J., Racero-Casarrubia, J., Humanez-López, E., \& Arias-Alzate, A. (2019). Predicting Greater Grison Galictis vittata presence from scarce records in the department of Cordoba, Colombia. Small Carnivore Conservation, 57, 34-44.

Hawley, T. (2016). Common frogs of the Osa Peninsula, Costa Rica. https://bit.ly/3rxEe99

Herra, L., \& Castañeda, F. (2014). Greater grison (Galictis vittata, Schreber 1776) first cameratrap records in Honduras. En Sociedad Mesoamericana para la Biología y la Conservación (Eds.), Libro de Resúmenes XVIII Congreso de la Sociedad Mesoamericana para la Biología y la Conservación. Copán Ruinas, Honduras.

Hidalgo-Mihart, M.G., Juárez-López, R., Contreras-Moreno, F., De La Cruz, A.J., López-Luna, M.A., \& De La Cruz, Y.B. (2018). Greater grison (Galictis vittatta) hunts a Central American Indigo Snake (Drymarchon Melanurus) in 
Southeastern Mexico. The Southwestern Naturalist, 63(3), 197-199. https://doi.org/10.1894/0038-4909-63-3197

Hurtado, J., \& Soto, C. (2017). Manual para el Monitoreo Participativo de Vertebrados Terrestres a través de Cámaras Trampa en Costa Rica. Proyecto MAPCOBIO-SINAC.

Kays, R.W. (1996). Comments on the behaviour of a grison (Galictis vittata) hunting an agouti (Dasyprocta punctata). Small Carnivore Conservation, 15, 5.

Leenders, T. (2016). Amphibians of Costa Rica: a field guide. Cornell University Press.

Malavassi, L. (1991). I. Captura del Grisón (Gallictis vittata). II. Actividad del Grisón (Gallictis vittata) en cautiverio. III. Posible ciclo reproductivo del Grisón (Gallictis vittata) en Costa Rica [Tesis de Maestría,Universidad Nacional de Costa Rica].

Meza-Joya, F.L., Ramos, E., Cediel, F., Martínez-Arias. V., Colmenares, J. \& Cardona, D. (2018). Predicted distributions of two poorly known small carnivores in Colombia: the Greater Grison and Striped Hog-nosed Skunk. Mastozoología Neotropical, 25(1), 89-105. https://doi.org/10.31687/saremMN.18.25.1.0.09

Rodríguez, F.J. \& Chinchilla, F. (1996). Lista de mamíferos de Costa Rica. Revista Biología Tropical, 44 (2), 877-890.

Sáenz-Bolaños, C., Alfaro, L.D., Carrillo, E. (2009). Presencia de Galictis vittata (Carnivora: Mustelidae) en el Caribe Sur y Pacífico Norte de Costa Rica. Brenesia, (71-72), 71-72.

Sistema Nacional de Áreas de Conservación [SINAC]. (2017). R-SINAC-CONAC-092-2017. Lista oficial de especies en peligro de extinción y con poblaciones reducidas y amenazadas. San José, Costa Rica: Diario Oficial La Gaceta.

Sunquist, M.E., Sunquist, F., \& Daneke, D.E. (1989). Ecological separation in a Venezuelan Llanos carnivore community. En Redford, K.H., \& Eisenberg, J.F. (Eds.), Advances in neotropical mammalogy, (pp. 197-232). Sandhill Crane Press.

Timm, R.M., Wilson, D.E., Clauson, B.L., Laval, R.K., \& Vaughan, C.S. (1989). Mammals of the La Selva-Braulio Carrillo complex, Costa Rica. North American Fauna, 75(75), 1-162. https://doi.org/10.3996/nafa.75.0001

Yesen, E., \& Tarifa, T. (2003). Galictis vittata. Mammalian Species, (727), 1-8. https://doi.org/10.1644/0.727.1 